\title{
COVID-19: Risks to Pregnancy and Immunity between Mother and Fetus
}

\author{
Nada M A Hashem* \\ Department of Physiology, Faculty of Veterinary Medicine, Mansoura University, Egypt
}

*Corresponding author: Nada Mohamed Ali Hashem, Department of Physiology, Faculty of Veterinary Medicine, Mansoura University, Egypt.

\section{ARTICLE INFO}

Received: 㗀 January 18, 2021

Published: 蔧 January 27, 2021

Citation: Nada M A Hashem. COVID-19: Risks to Pregnancy and Immunity between Mother and Fetus. Biomed J Sci \& Tech Res 33(3)-2021. BJSTR. MS.ID.005403.

Keywords: SARS COVID-19; Pregnancy; Fetus; Immunity

\section{ABSTRACT}

There is no doubt that the immune system of a pregnant mother plays a very important role in the establishment, maintenance, and completion of a healthy pregnancy. Immune cells and cytokines play an important role in the acceptance and development of the fetus by the maternal immune system. A successful pregnancy relies on resistance against any infection and tolerance of the immune system of the mother toward the fetus. Infection during pregnancy may lead to premature delivery, abortion, growth defects, neonatal death, and other adverse outcomes. The COVID-19 has changed lives across the world and these changes that occurred have a psychological effect for all people especially for pregnant women who worry about their pregnancy more than before. The risks of infection of pregnant women and the outcomes of this COVID-19. 19 infection on fetus and mother become a major problem. This review will be based on recently published literature and official documents to demonstrate the effect of COVID-19 on pregnancy and its risks. This review shows a brief to clinical features of COVID-19 and its spreading all over the world with a demonstration of the role of the immune system during pregnancy and against any infection. Also, COVID-19 effect on pregnant women and fetus with its outcomes and how to manage and treat a case of COVID-19 pregnant patient. This review demonstrates pregnant women infected with SARS-CoV-2 in comparison with SARS-CoV and MERS-CoV with their complications. Finally, the scientific research and articles on pregnancy and COVID-19 are insufficient despite papers and documents published on pregnancy and COVID-19. So, fetus and pregnant mother infected with COVID-19 need special attention for the prevention, diagnosis, and management of COVID-19.

Abbreviations: ALI: Acute Lung Injury; ARDS: Acute Respiratory Distress Syndrome; ACO: American College of Obstetricians and Gynecologists; CD 16: A Molecule found on the surface of Leukocyte cell; CD56: Marker of natural killer Cells and expressed by Immune cells; CDC: Centers for Disease Control and Prevention; COVID 19: Coronavirus 2019; CT: A Computerized Tomography Scan; FIRS: Fetal Inflammatory Response Syndrome; H1N1: Influenzas A Virus; H5N1: A Type of Influenza virus called Avian Influenza; IL1: Interleukin 1; IL6: Interleukin 6; IL12: Interleukin 12; ISUOG: The International Society of Ultrasound in Obstetrics \& Gynecology; MERS-COV: Middle East Respiratory Syndrome Coronavirus; MMPS: Matrix Metalloproteinases; NK CELLS: Natural Killer Cells; PRRS: Pattern Recognition Receptor; QRTPCR: Quantitative Polymerase Chain Reaction; RCOG: Royal college of Obstetricians and Gynecologists; RNA: Ribonucleic Acid; SARS: Severe Acute Respiratory Syndrome; SARS COV2: Severe Acute Respiratory Syndrome Coronavirus; SMFM: The Society for Maternal-Fetal Medicine; TH1: T helper 1; TH2: T helper 2; TH17: T helper 17; TNF: Tumor Necrosis Factor; UK: United Kingdom; UNK CELLS: Uterine Natural Killer Cells; VEGF: Vascular Endothelial Growth Factor; WHO: World Health Organization

\section{Introduction}

\section{COVID-19}

Coronaviruses contains A simple-stranded RNA as a nucleic material, its whole is ranging from 26 to $32 \mathrm{kbs}$ in length and its diameter is $65-125 \mathrm{~nm}$ (Figure 1) by [1]. Coronaviruses subgroups are alpha (a), beta (b), gamma (c), and delta (d) coronavirus. Many diseases cause acute lung injury (ALI) and acute respiratory distress syndrome(ARDS), pulmonary failure and may be fatal as severe acute 
respiratory syndrome coronavirus (SARS-CoV), H5N1 influenza A, H1N1 2009, and Middle East respiratory syndrome coronavirus (MERS-CoV). These viruses were thought to infect only animals until the world witnessed a severe acute respiratory syndrome (SARS) outbreak caused by SARS-CoV, 2002 in Guangdong, China [2]. Only a decade later, another pathogenic coronavirus, known as Middle East respiratory syndrome coronavirus (MERS-CoV) caused an endemic in Middle Eastern countries [3,4] demonstrated that in December 2019, many diseased conditions with pneumonia appeared in Wuhan city in China and these cases suffered from many symptoms like weakness, dry cough and restlessness with high temperature reached to dyspnea in serious cases besides peripheral pulmonary plaques and interstitial lesions in chest scan. Liu et al., [5] showed that this virus which appeared in China and distributed to many countries was named severe acute respiratory syndrome coronavirus 2 (SARS-COV-2), which has a hereditary resemblance to SARS-CoV

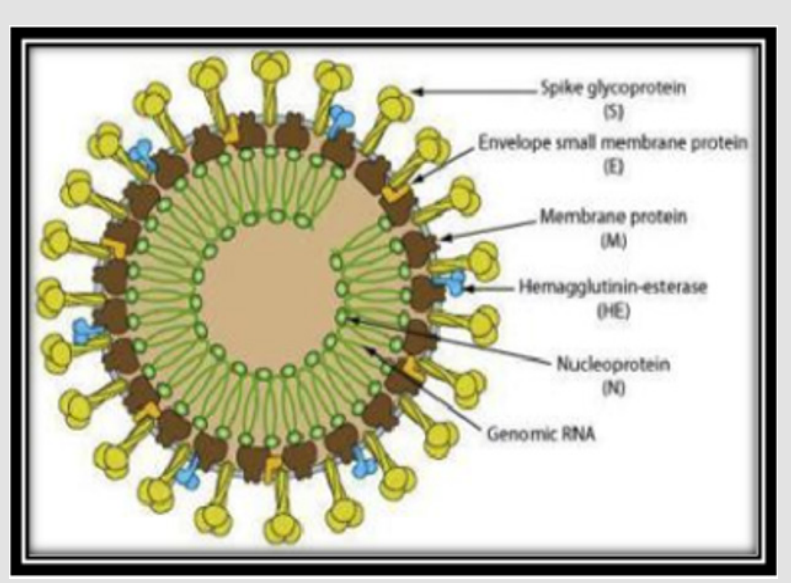

Figure 1: Coronavirus structure[1]

\section{COVID-19 Spreading}

WHO announced that MERS coronavirus causes infection to more than 2428 individuals and 838 deaths [6]. MERS-CoV is a beta-coronavirus subgroup and genetically varied from other human-CoV. The epidemic of MERS CoV started with simple flu like symptoms then progressed to all parts of the respiratory system and cause serious pneumonia. Similar to SARS coronavirus, patients diseased with MERS-coronavirus suffer pneumonia, followed by ARDS and kidney defects [7]. The virus was described as a unique coronavirus from the genetic sequence of patients isolates. Initially, [8] was indicated that the source of infection was the fish market or from diseased animals or birds in china. Besides, a survey revealed that the spreading of virus infection occurred from one person to another by direct contact with infected one exposed to sneezing, coughing, respiratory droplets, and these enter the lung through the nasal or oral passage.

\section{Pregnancy and Immunity}

[9] showed that the communication between mother and semi allogeneic fetus to placental protection of the fetus was due to expression of histocompatibility antigens in fetal tissues and regulation of maternal immunity for providing an immune tolerance environment for the fetus. At the time of placentation, immune cells count rise in uterine tissue 3-5 days after ovulation reached $25 \%-40 \%$ of uterine leukocytes before implantation and considered the highest number at early three months of pregnancy due to their functions in placentation and protection against infection [10]. The uterine killer cells are different from peripheral killer cells in their genetic structure and action. They are revealed a large number of (CD56), but they lack expression of CD16 which present on most peripheral natural killer cells [10]. Peripheral CD56 and CD16+ NK cells were considered killer cells as they interfered with both natural and specific antibody killer, whereas uNK cells are faint cytotoxic and do not attack trophoblast cells in normal conditions [11]. In addition, uNK cells produce a high number of immunomodulatory cytokines, [12] matrix metalloproteinases (MMPs), [13], and angiogenic factor [14]. In a healthy pregnancy, macrophages cells are found in decidua before the presence of trophoblast and play a role in blood supply by producing (MMP9) that make tissue remodeling and (vascular endothelial growth factor [VEGF]) for angiogenesis [15].

\section{Pregnant Mother Immunity and Infection}

Mor et al. [16] said that pregnancy is a period of high risk for infection and her maternal immune system is responsible for defending against infectious microbes because the fetus and placenta responses are restrained Jamieson et al., [17] showed that the main target of the immune system during pregnancy is protecting both mother and fetus from viral and microbial infections. The suppression of pregnant mother cell-mediated immunity turned the Th1 to the Th2 immunity and rise the perceptivity to any disease [18]. However, Zhang et al., [19] showed that certain diseased cases among pregnant patients have bad outcomes ranged from symptoms to death which differ between developed and non-developed countries as in the US or Canada, pregnant women with varicella are better than those diagnosed in underdeveloped countries. Brown and Derkits, [20] demonstrated that children of infected mothers during pregnancy have significantly higher frequencies of neurological disorders as schizophrenia and autism spectrum disorders. The reason for these disorders is due to activation of the maternal inflammatory immune responses reviewed by [21,22]. Studies in rodents proved by clinical findings confirmed rat models have disorders from immune system activation [23]. Mandal et al., [24] showed that pregnant mother immunity enhances the brain and the immune system development in the offspring due to secretion of interleukin 1, interleukin6 and interleukin 12. In addition to granulocyte-macrophage colony-stimulating factor and tumor necrosis factor-alpha in the blood of pregnant mothers and secreted in amniotic fluid surrounding the fetus.

Mandal et al., [25] showed that fetuses have a proinflammatory response and high levels of $\mathrm{T}$ helper1, $\mathrm{T}$ helper17, and cytotoxic $\mathrm{T}$-cell due to stimulation of the immune system of their mothers. 
Infection is very dangerous during pregnancy not only on the mother but also on the fetus. Clinical studies showed that during placental infection, concentrations of Interleukin 1, 6, 8, and TNF- $\alpha$ increased and fetal this called fetal inflammatory response syndrome (FIRS) [26]. However, any infection that enters the placenta stimulates a rapid immune response that leads to uterine infection. [27] and this is caused by activation of pattern recognition receptors and high secretion of interleukin 1 (IL1) and TNF- $\alpha$ [28]. The result of the infection affects both mother and fetus as poor pregnancy may lead to fetal deformities, decrease fetal weight and early parturition [29].

\section{COVID-19 and Pregnancy}

The physiological changes and stress which occurred during pregnancy cause changes in immune, cardiovascular, and respiratory systems [30]. [31] showed that viral infections that affect the respiratory system during pregnancy may lead to birth defects as low birth weight and preterm birth. Viruses can get congenitally transmitted to the fetus such as Ebola, Cytomegalovirus, Zika, and Rubella, most of these viruses enter the fetus through the hematogenous route in which the virus circulating in the mother's bloodstream reaches and enters the placenta through the chorionic villous tree and reached fetal blood vessels. [32] Clinical data of the SARS epidemic showed that from 12 SARS-COV infected pregnant women, 2 had intrauterine growth problems in the 2 nd and 3rd trimester and 3 died during pregnancy [33]. [34] showed that during the MERS-CoV epidemic, $91 \%$ of 11 pregnant infected females had problems in pregnancy outcomes such as premature delivery, maternal and perinatal death and there was not one case to suggest the intrauterine transmission of the MERS virus. [35] showed that clinical symptoms of patients infected with SARS and MERS ranged from no symptoms to severe disease and death and 80\% of COVID-19 patients in hospitals having cough and fever. [36] showed that the COVID-19 pandemic is spreading around the world and Pregnant women most likely to be affected and most clinical findings of covid-19 pregnant patients are delivered early and by cesarean section.

[35] showed that symptoms at the onset of COVID-19 infection were reported for 35 pregnant women (69\%), and the symptoms were similar to those described in nonpregnant patients (Table 1). Fever was presented in 17 pregnant women (48\%) in addition to 16 women (46\%) who suffered from dry cough and this symptom may be alone or with another symptom. Other 8 infected patients had fever only in the postpartum period with $23 \%$. Other symptoms included fatigue (three cases), myalgia (three cases), sore throat (five cases), dyspnea (four cases), diarrhea (two cases), malaise (two cases), and two cases of cholecystitis. There was a case of hypertension during pregnancy at 27 weeks of pregnancy and a case of preeclampsia at 31 weeks of pregnancy and in these two cases, symptoms appeared after COVID-19 diagnosis [35].
Table 1: Reported symptoms at diagnosis of infected COVID-19 pregnant women.

\begin{tabular}{|c|c|}
\hline Onset symptoms & $\begin{array}{c}\text { Reported only for 35 of 51 patients, } \\
\text { often in combination (\%) }\end{array}$ \\
\hline Dry cough & $16 / 35(45.7)$ \\
\hline Fever admission & $17 / 35(48.6)$ \\
\hline Postpartum fever & $8 / 35(22.9)$ \\
\hline Myalgias & $3 / 35(8.6)$ \\
\hline Malaise & $2 / 35(5.7)$ \\
\hline Dyspnea & $4 / 35(11.4)$ \\
\hline Sore throat & $5 / 35(14.3)$ \\
\hline Diarrhea & $2 / 35(5.7)$ \\
\hline Fatigue & $3 / 35(8.6)$ \\
\hline Cholecystitis & $2 / 35(5.7)$ \\
\hline
\end{tabular}

Pregnancy Outcomes in COVID-19 Infected Females in China

A study reviewed by [5] on COVID-19 pregnant patients at the period from 20 January 2020 to 10 February 2020 showed pneumonia in their lung by CT examination. Fifteen COVID-19 positive pregnant women had many symptoms as fever in 13 women out of 15 , cough in 9 women out of 15 pregnant patients, and lymphocytopenia in 12 out of 15 pregnant patients. Eleven out of fifteen women had successful labor (ten cesarean cases and one normal labor). The newborn babies did not have SARS-COV- 2 and no cases of abortion or fetal asphyxia or death. However, [37] showed that in Wuhan Children's Hospital 33 newly born baby born to mothers with COVID-19 by caesarian section due to maternal pneumonia and stress, four out of 33 showed breath problems and nasopharyngeal and anal swabs of the other three neonates tested positive SARS-CoV-2 on days 2 and 4 but negative on days 6-7 of their lives. All these COVID-19 neonates had fever and pneumonia and they were treated with antibiotics and proper ventilation. SARS $\mathrm{CoV}-2$ source in the neonates may be from mother to the neonates during pregnancy. World Health Organization (WHO) has reported that women of the same reproductive age have no diversity in symptoms between nonpregnancy state and pregnancy state [38].

However, [39] demonstrated that physiological changes that occurred to pregnant women altered the immune response and so their response to covid-19 resemble their response to other viral infections. [40] showed that there are no maternal risks, such as covid-19 infection after parturition and early parturition. In contrast, [41] reported that both mother and fetus suffered from problems as early parturition, breathing problem, and fetal death. Additionally, [42] showed one case with maternal death and one case with intrauterine fetal death at the last three months of pregnancy infected with COVID-19 in Iran. [43] showed nine pregnant women infected with severe COVID-19 at the end of the gestation period. Seven out of nine women died, one out of 9 
women still sick and ventilator-dependent and only one case out of nine women recovered after a long time of treatment. Moreover, [44] announced a clinical finding of abortion in the half of gestation period in a woman positive COVID-19, but the clinical finding of samples obtained from amniotic fluid, cord blood, genital swabs, and breast milk, and the neonatal throat swabs showed negative for COVID-19 [45]. In contrast, [46] have reported a study that considered the first study showed that 3 swabs taken from 11 placentae of SARS-COV2 infected women were positive for SARS COV-2. This result increases the prospects of vertical transmission or intrapartum viral exposure and so clinical studies recommended that mothers have Cov-2 must be delivered by caesarian section to decrease exposure to tissues and thus decrease capabilities of vertical transmission.

\section{COVID-19 and Fetus}

[39] mentioned that a small number of clinical cases which determined the relationship between infected mother with SARS$\mathrm{CoV}-2$ and their newborn and the rate of intrauterine vertical transmission. [5] demonstrated that more research will be needed for the determination of vertical transmission and Fetal outcomes and problems from infected mothers with COVID-19 and its effect on fetal breath and time of parturition. [40] reported that a clinical study of 7 pregnant mothers positive for SARS COV2 and after their caesarian sections, the newborn babies were suspected for COVID-19 and so they tested, and one baby was recognized suffering from respiratory problems and tested positive for COVID-19. The baby symptoms were regularly managed with specific treatment till complete recovery [41,47] reported a clinical finding of two babies suffering from lymphopenia and mild pneumonia, with no other symptoms, and they were born from an infected mother with COVID-19. [48] supported the previous data with a case of a suspected newly born baby from an infected mother that have no symptoms, but a clinical blood test showed lymphopenia with confused liver function tests. Liu et al., [5,42] announced many fetal deaths, where opposed pregnancy characteristics including severe mothers state with multiple damaged organs lead to bad fetal outcomes.

\section{Protocols and Guidelines for COVID-19 During and after Pregnancy}

World Health Organization [49] approved guidelines for the treatment of pregnant patients. The most current guidelines, developed on April 22, 2020, by 9 papers from 6 societies: SMFM, ACOG, RCOG, ISUOG, CDC, and WHO (Figure 2). Pregnant patients should be tested for symptoms, history, contact history, who meets and who visits and who has any symptoms should be tested for SARS-CoV-2 using qRT-PCR. The birth should take place by following specific indications and precautions, with taking care of the clinical symptoms of mothers and treating these symptoms as required and WHO organization and other Societies recommended that healthy asymptomatic individual must provide support to mother during parturition. The delivery time and method should be chosen according to the severity of infection, patient history, gestational age and fetal conditions. Mothers and newly born babies should be room-in and breastfeed, with following up precautions and guidelines of WHO and the UK Royal College of Obstetricians and Gynecologists. Babies from COVID-19 infected mothers should undergo a careful examination, and their temperature, rate of respiration, and heartbeats should be regularly examined. Mothers should follow to wear masks during breastfeeding. Mothers must wash their hands before touching anything and should avoid coughing while their babies are feeding. All surfaces and breast pumps must be sanitized after each use. Mothers should take care of hand hygiene and disinfection, wearing N-95 masks during breastfeeding [49].

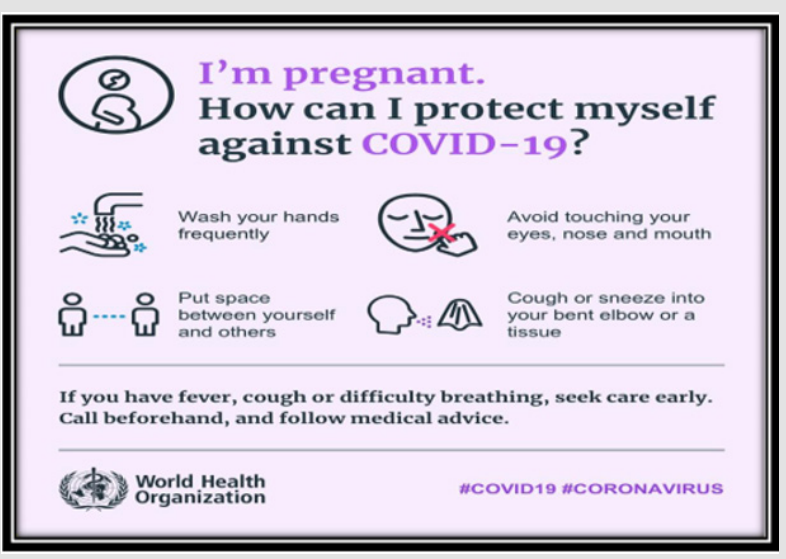

Figure 2: Guidelines of WHO for pregnant mother [49].

\section{Conclusion}

The immune system has a vital role in providing the proper growth of the fetus with normal placentation in a healthy manner from the first time of fertilization till parturition. The immune physiological changes increase the chance of covid-19 infection during the gestation period. During gestation, pregnant mothers should protect themselves from exposure to any contagion or epidemic during pregnancy and keep away from crowded places and gatherings. In addition, future research and more investigation is needed to cover more and more on COVID-19 influence on pregnant mothers and fetuses. Histopathological examination is needed for more explanation of covid-19 and its action on the placenta and uterus during pregnancy.

\section{References}

1. De Haan C, L Kuo, PS Masters, H Vennema, PJM Rottier (1998) Coronavirus Particle Assembly: Primary Structure Requirements of the Membrane Protein. J Virol 72(8): 6838-6850.

2. Zhong NS, BJ Zheng, YM Li Poon, ZH Xie, KH Chan, et al. (2003) Epidemiology and cause of severe acute respiratory syndrome (SARS) in Guangdong, People's Republic of China, in February 2003. Lancet 362(9393): 1353-1358.

3. Wang N, X Shi, L Jiang, S Zhang, D Wang, et al. (2013) Structure of MERSCoV spike receptor-binding domain complexed with human receptor DPP4. Cell Res 23(8). 
4. (2020a) WHO, Novelcoronavirus- China. China.

5. Liu D, L Li, X Wu, D Zheng, J Wang (2020) Pregnancy and perinatal outcomes of women with coronavirus disease (COVID-19) Pneumonia: A preliminary analysis. Am J Roentgenol 215(1): 127-132.

6. Rahman A, A Sarkar (2019) Risk factors for fatal Middle East respiratory syndrome coronavirus infections in Saudi Arabia: Analysis of the WHO Line list, 2013-2018. Am J Public Health 109(9): 1288-1293.

7. Memish ZA, AI Zumla, RF Al Hakeem, AA Al Rabeeah, GM Stephens (2013) Family Cluster of Middle East Respiratory Syndrome Coronavirus Infections. N Engl J Med 368: 2487-2494.

8. Riou J, CL Althaus (2020) Pattern of early human-to-human transmission of Wuhan 2019 novel coronavirus (2019-nCoV), December 2019 to January 2020. Euro surveillance 25(4): 2000058.

9. Billingham RE, L Brent, PB Medawar (1953) Actively acquired tolerance of foreign cells. Nature 172(4379): 603-606

10. King A (2000) Uterine leukocytes and decidualization. Hum Reprod Update 6(1): 28-36.

11. King A, C Birkby, YW Loke (1989) Early human decidual cells exhibit NK activity against the K562 cell line but not against first trimester trophoblast. Cell Immunol 118(2): 337-344.

12. Koopman LA, HD Kopcow, B Rybalov, JE Boyson, JS Orange (2003) Human Decidual Natural Killer Cells Are a Unique NK Cell Subset with Immunomodulatory Potential. J Exp Med 198(8): 1201-1212.

13. Naruse K, GE Lash, BA Innes, HA Otun, RF Searle, et al. (2009) Localization of matrix metalloproteinase (MMP)-2, MMP-9 and tissue inhibitors for MMPs (TIMPs) in uterine natural killer cells in early human pregnancy. Hum Reprod 24(3): 553-561.

14. Lash GE (2006) Expression of angiogenic growth factors by uterine natural killer cells during early pregnancy. J Leukoc Biol 80(3): 571-580.

15. Faas MM, F Spaans, P De Vos (2014) Monocytes and macrophages in pregnancy and pre-eclampsia. Front Immunol 5: 298.

16. Mor G, I Cardenas (2010) The Immune System in Pregnancy: A Unique Complexity. Am J Reprod. Immunol 63(6): 425-433.

17. Jamieson DJ, RN Theiler, SA Rasmussen (2006) Emerging infections and pregnancy. Emerg Infect Dis 12(11): 1638-1643.

18. Muzzio D, M Zygmunt, F Jensen (2014) The role of pregnancy-associated hormones in the development and function of regulatory B cells. Front Endocrinol (Lausanne) 5: 39.

19. Zhang HJ, V Patenaude, H Abenhaim (2014) Maternal Outcomes in Pregnancies Affected by Varicella Zoster Virus Infections. Obstet Gynecol pp. 123.

20. Brown AS, EJ Derkits (2010) Prenatal infection and schizophrenia: A review of epidemiologic and translational studies. Am J Psychiatry 167(3): 261-280

21. Jonakait GM (2007) The effects of maternal inflammation on neuronal development: possible mechanisms. Int J Dev Neurosci 25(7): 415-425.

22. Patterson PH (2011) Maternal infection and immune involvement in autism. Trends Mol Med 17(7): 389-394.

23. Smith SEP, J Li, K Garbett, K Mirnics, PH Patterson (2007) Materna immune activation alters fetal brain development through interleukin-6. J Neurosci 27(40): 10695-10702.

24. Mandal M, AC Marzouk, R Donnelly, NM Ponzio (2011) Maternal immune stimulation during pregnancy affects adaptive immunity in offspring to promote development of TH17 cells. Brain Behav Immun 25(5): 863871.

25. Mandal M, R Donnelly, S Elkabes, P Zhang, D Davini, et al. (2013) Maternal immune stimulation during pregnancy shapes the immunological phenotype of offspring. Brain Behav Immun 33: 33-45.
26. Racicot K, JY Kwon, P Aldo, M Silasi, G Mor (2014) Understanding the complexity of the immune system during pregnancy. Am J Reprod Immunol 72(2): 107-116.

27. Kraus TA, SM Engel, RS Sperling, L Kellerman, Y Lo, et al. (2012) Characterizing the pregnancy immune phenotype: Results of the viral immunity and pregnancy (VIP) study. J Clin Immunol 32(2): 300-311.

28. Raj RS, EA Bonney, M Phillippe (2014) Influenza, immune system, and pregnancy. Reprod Sci 21(12): 1434-1451.

29. Rogerson SJ, L Hviid, PE Duffy, RF Leke, DW Taylor (2007) Malaria in pregnancy: pathogenesis and immunity. Lancet Infect Dis 7(2): 105-117.

30. Yang H, C Wang, LC Poon (2020) Novel coronavirus infection and pregnancy. Ultrasound Obstet Gynecol 55(4): 435-437.

31. Rasmussen SA, DJ Jamieson, TM Uyeki (2012) Effects of influenza on pregnant women and infants. Am J Obstet Gynecol 207(3 suppl): S3-S8.

32. Siston AM, SA Rasmussen, MA Honein, AM Fry, K Seib, et al. (2010) Pandemic 2009 influenza A (H1N1) virus illness among pregnant women in the United States. Jama 303: 1517-1525.

33. Chan JF, WS Yuan, KH Kok, KKW To, H Chu, et al. (2020) A familial cluster of pneumonia associated with the 2019 novel coronavirus indicating person-to-person transmission: a study of a family cluster. Lancet 395(10233): P514-523.

34. Alfaraj SH, J Aal Tawfiq, ZA Memish (2019) Middle East Respiratory Syndrome Coronavirus (MERS-CoV) infection during pregnancy: Report of two cases \& review of the literature. J Microbiol Immunol Infect 52(3): 501-503.

35. Chen H, J Guo, C Wang, F Luo, X Yu, et al. (2020) Clinical characteristics and intrauterine vertical transmission potential of COVID-19 infection in nine pregnant women: a retrospective review of medical records. Lancet 395(10226): 809-815.

36. Avram CM, KS Greiner, E Tilden, AB Caughey (2020) Additional Considerations Regarding Point-of-care HIV Viral Load in Pregnant Women Without Prenatal Care. Am J Obstet Gynecol.

37. Zeng L, S Xia, W Yuan, K Yan, F Xiao, et al. (2020) Neonatal EarlyOnset Infection with SARS-CoV-2 in 33 Neonates Born to Mothers with COVID-19 in Wuhan, China. JAMA Pediatr 174(7): 722-725.

38. WHO, W(PRC) Aylward, Bruce (WHO) Liang (2020) Report of the WHOChina Joint Mission on Coronavirus Disease 2019 (COVID-19). WHOChina Jt. Mission Coronavirus Dis 2019

39. Mullins E, D Evans, RM Viner, P O Brien, E Morris (2020) Coronavirus in pregnancy and delivery: rapid review. Ultrasound Obstet. Gynecol 55(5): 586-592.

40. Yu N, W Li, Q Kang, Z Xiong, S Wang, et al. (2020) Clinical features and obstetric and neonatal outcomes of pregnant patients with COVID-19 in Wuhan, China: a retrospective, single-centre, descriptive study. Lancet Infect Dis 20(5): 559-564

41. Zhu H, L Wang, C Fang, S Peng, L Zhang, et al. (2020) Clinical analysis of 10 neonates born to mothers with 2019-nCoV pneumonia. Transl Pediatr 9(1).

42. Karami P, M Naghavi, A Feyzi, M Aghamohammadi, MS Novin, et al (2020) Mortality of a pregnant patient diagnosed with COVID-19: A case report with clinical, radiological, and histopathological findings. Travel Med Infect Dis: 101665.

43. Hantoushzadeh G, SA Shamshirsaz, AA Aleyasin, AD Seferovic, MD Aski, et al. (2020) Journal Pre-proof Maternal Death Due to COVID-19 Disease. Am J Obstet Gynecol 223(1): 109e1-109e16.

44. Baud D, G Greub, G Favre, C Gengler, K Jaton, et al. (2020) SecondTrimester Miscarriage in a Pregnant Woman with SARS-CoV-2 Infection. JAMA. J Am Med Assoc 323(21): 2198-2200.

45. Li N, L Han, M Peng, Y Lv, Y Ouyang, et al. (2020) Maternal and Neonatal Outcomes of Pregnant Women with Coronavirus Disease 2019 
(COVID-19) Pneumonia: A Case-Control Study. Clin Infect Dis 71(16) 2035-2041.

46. Penfield CA, SG Brubaker, MA Limaye, J Lighter, AJ Ratner, et al. (2020) Detection of SARS-COV-2 in Placental and Fetal Membrane Samples. Am J Obstet Gynecol MFM 2(3): 100133.

47. Fan C, D Lei, C Fang, C Li, M Wang, et al. (2020) Perinatal Transmission of COVID-19 Associated SARS-CoV-2: Should We Worry? Clin Infect Dis: 226.

ISSN: 2574-1241

DOI: 10.26717/BJSTR.2021.33.005403

Nada M A Hashem. Biomed J Sci \& Tech Res

(C) (i) This work is licensed under Creative

Submission Link: https://biomedres.us/submit-manuscript.php
48. Wang X, Z Zhou, J Zhang, F Zhu, Y Tang, et al. (2020) A case of 2019 Novel Coronavirus in a pregnant woman with preterm delivery. Clin Infect Dis 71(15): 844-846

49. (2020b) WHO, Q\&A on COVID-19, pregnancy, childbirth, and breastfeeding, WHO.

$\begin{array}{ll}\text { BIOMEDICAL } & \text { Assets of Publishing with us } \\ \text { RESEARCHES } & \text { - Global archiving of articles } \\ \text { - Immediate, unrestricted online access } & \text { - Rigorous Peer Review Process } \\ & \text { - Authors Retain Copyrights } \\ \end{array}$

